\title{
User Personas: Discovering Factors That Influence Genz's Sedentary Lifestyle for the Development of a Mobile Exergame Design Model
}

\author{
Hasdina Lynn Hashim ${ }^{1}$, Azrina Kamaruddin², Azrul Hazri Jantan ${ }^{3}$, Puteri Suhaiza Sulaiman ${ }^{4}$ \\ ${ }^{1,2,3,4}$ Faculty of Computer Science \& Information Technology, Universiti Putra Malaysia, \\ 43400 UPM Serdang, Selangor, Malaysia \\ hlhashim@outlook.com ${ }^{1}$
}

Article History: Received: 11 October 2020; Accepted: 27 December 2020; Published online: 8 February 2021

\begin{abstract}
The use of mobile devices for youths in tertiary education is found to be linked to sedentary behaviour. This paper attempts to create user personas on these youths and finding the factors that affect their sedentary lifestyles, which could assist the development of a mobile exergame design model. An online survey consisting of sociodemographic and IPAQ questionnaires was used to collect data on students in a public university. Three user personas were derived, which are based on their weekly physical activity levels: Low, Moderate, and High. It was found that income status, time management, daily smartphone usage, and fitness self-rating has a direct relationship with physical activity levels. The user personas share a common factor in that smartphone usage is essential to manage the youths' daily activities regardless of their physical activities. The derived user personas would assist future mobile exergame design model development by concentrating on the influencing factors from the Low and Moderate physical activity level groups.
\end{abstract}

Keywords: Generation Z, IPAQ, exergame, user persona, mobile exergame design model

\section{Introduction}

The sedentary lifestyle affects more than sixty percent of the Malaysian adult population (Lian, Bonn, Han, et al., 2016) with obesity being a major risk factor for many cardiovascular diseases such as coronary heart rate disease, heart failure, and stroke (Swift, Johannsen, Lavie, et al., 2014). The use of smartphones by youths in tertiary education is directly linked to sedentary behaviour; they are less active and less intensive with their exercises when engaged with their smartphones (Barkley, Lepp, \& Salehi-esfahani, 2016).

This paper aims to recognise the sedentary lifestyle of Malaysian youths currently in tertiary education, in terms of their smartphone usage and physical activity. This, in turn, could assist in developing a mobile exergame design model suited for them. The research is done via an online survey, and the collected data aims to answer the following research questions (RQ):

- RQ1: What kind of user personas can we identify based on these youths' weekly physical activity; categorised in High, Moderate, and Low physical activity users?

- RQ2: What are the factors that influence and motivate each user persona that affects these youths' sedentary lifestyle?

- $\quad$ RQ3: How could the user personas be used to assist the development process of a mobile exergame design model for youths in tertiary education?

\section{Literature Review}

\subsection{Generation $\mathrm{Z}$}

Youths currently enrolled in their tertiary studies, e.g., university undergraduates, were born from the mid1990s; the top part of the generation gap commercially known as Generation Z (GenZ) (Piette, List, Rana, et al., 2015). GenZs are exposed to the internet since young and adept with technology devices such as smartphones and tablets (Ozkan \& Solmaz, 2015) and (Hussain et al., 2019). Daily tasks are managed at their fingertips on the go, and social media applications (apps) such as Instagram and TikTok play prominent roles in their daily interactions. Smartphone usage is common with GenZs, and its utilisation (be it for work or entertainment) is generally conducted when sedentary. When it comes to physical activity (PA), certain factors affect GenZs' motivation to exercise. According to (Biber, 2013), GenZs are motivated to be involved in PA when there are high attraction factors: being active and healthy, being able to progressively master skills, getting accomplishment recognised, and interacting and supporting one another socially. In contrast, GenZs are aversed to doing PA when there are possibilities of being incompetent, fear of injury, and lack of social support (T. Padmapriya et al, 2020). 


\subsection{Sedentary Behaviour and Physical Activity}

Sedentary behaviour (SB) is defined as any waking behaviour, characterised by an energy expenditure of less than or equal to 1.5 metabolic equivalent task (MET) while in a sitting or reclining posture (Network, 2013). MET is used to represent the intensity of a PA by measuring the amount of oxygen consumed, and calories burn at rest (Júdice, Hamilton, Sardinha, et al., 2016). The MET values of activities such as Light PA (walking) is between 1.5 to 3.0 METs, Moderate PA (walking at a brisk pace) is between 3.0 to 6.0 METs and Vigorous PA (jogging or running) is above 6.0 METs (Haskell, Lee, Pate, et al., 2007). To promote and maintain health, healthy adults need to perform moderate PA (MPA) for a minimum of 150 minutes a week or perform vigorous PA (VPA) for a minimum of 60 minutes a week (Garber, Blissmer, Deschenes, et al., 2011).

\subsection{Exergame Design Model}

In the digital environment, exergame (a portmanteau of exercise and game) is an interactive digital game that utilises physical exertion as the determinant in reaching the game's goal (Mueller, Khot, Gerling, et al., 2016). Exergame can be used to meet the recommended weekly PA, and it is also available on mobile devices such as smartphones, thus mobilising the exergame experience (Nigg, Mateo, \& An, 2017). Pokemon Go is a type of mobile exergame as it indirectly encourages PA to reach game's goals (Marquet, Alberico, \& Hipp, 2018).

A design model (DM) provides a visual representation or guide on the conduct of the research study. In the exercise or exergame context, a DM can document the changes that affect players' psychological and physiological states. Self-determined Theory, Flow Model, Game Flow Model, Dual-Flow model (DFM), ISCAL model, and Pleasurable Persuasive Model (PPM) are a few of the existing models used extensively in PA research on exercise or exergame (Hashim, Kamaruddin, Wirza Rahmat, et al., 2018) and (Hussain et al,. 2016). A conceptual mobile exergame design model was proposed, adapted from the DFM and PPM (Hashim, Kamaruddin, \& Jantan, 2019).

\subsubsection{Understanding Users}

Previous researches on exergame DM hardly addressed understanding the users, thus lacking any existing user maps to global ideals (Han, Pan, Zhang, et al., 2016). User participations are only noted for product evaluation (Wang, Hagen, \& Høivik, 2018). A specific domain such as the Malaysian GenZs have different lifestyles in comparison to global users. Thus an input from the Asian perspective may address any research gaps.

User-centred design (UCD) is an iterative design process that has four distinct phases in its operation:Discovering Requirements, Designing Alternatives, Prototyping, and Evaluating; before a final product is released. UCD emphasised users' involvement from the beginning throughout the entire process until the end to ensure the final product is built around their requirements and needs (Sharp, Rogers, \& Preece, 2019). The first phase of the UCD - 'Discovering Requirements' is achieved by conducting user research. A user persona could then be created from the user research.

User persona (UP) is a character representation that could assist the product development with users in mind (Pruitt \& Adlin, 2006). Typically a few UPs are derived to describe each persona's background, motivations, and expectations. To answer our research questions, a UCD-centric approach was conducted in this study.

\section{Methods}

In this study, the user data was collected through an online survey via Google Forms. All consented respondents were between 18 to 25 years old, and each had a smartphone with internet connectivity to access the survey. All participants were undergraduates of the Universiti Putra Malaysia (UPM), with the majority being students of the Faculty of Computer Science and IT. The survey was accessible for four days from the 7th to the 10th of November 2019. A total of 35 respondents participated in the survey. The survey was a combination of two different collections of questionnaires that queried for data on sociodemographic profile (age, gender, race, income, smartphone usage) and health status (PA level and average sitting time among respondents). The latter was retrieved and extracted from a pre-validated, self-administered questionnaire: the International Physical Activity Questionnaire (Craig, Marshall, Sjöström, et al., 2003). 


\subsection{International Physical Activity Questionnaire (IPAQ)}

The purpose of the IPAQ is to obtain data on PA and has been validated for use in both developed and developing countries. There are two versions of the questionnaire; the short and long versions. For this study, the short version was selected due to its practicality and reliability (Craig, Marshall, Sjöström, et al., 2003). The IPAQ is used to calculate the amount of PAs done during a specified time frame and the amount categorised to pre-determined PA levels. The different PA levels can be used to create distinct UPs when combined with the sociodemographic profile data.

To determine each participant's PA level, the scoring protocol for IPAQ as per its guideline was used for analysis (Craig, Marshall, Sjöström, et al., 2003). A continuous PA score in the last 7 days for each participant is expressed in MET-minutes per week (MET-minutes/week), taking into account the frequency, intensity, and duration of the activities performed. These continuous scores were then categorised according to WHO classification for PA levels: High, Moderate, or Low (National Health and Morbidity Survey, 2015), as stated in Table 1.

Table 1. Physical Activity (PA) Levels

\begin{tabular}{|l|l|l|}
\hline Category & Level & Classification criteria \\
\hline 1 & High & Total PA of at least 3,000 MET-minutes/week \\
\hline 2 & Moderate & Total PA of at least 600 MET-minutes/week \\
\hline 3 & Low & do not meet criteria for Categories 1 or 2 \\
\hline
\end{tabular}

\section{Results and Discussions}

\subsection{Results}

Thirty-five respondents participated in the survey. The mean age of the respondents was 20.11 years old, with almost half of them being female (49\%). The mean monthly household income was RM4,542,86. The majority were from the Malay ethnic race (74\%). Most did exercise during their leisure time (71\%). A majority of them had played mobile games on their smartphones (83\%). Their mean daily smartphone usage was 5.77 hours, and the majority used the Android operating system (74\%). The primary use of the smartphone was for communication and social media (66\%). Refer to Table 2 for full data.

Table 2. Distribution of respondents' sociodemographic variables, smartphone usage and fitness engagement

\begin{tabular}{|l|l|}
\hline Variables & Mean \\
\hline Age & 20.11 \\
Daily smartphone use (hours) & 5.77 \\
Monthly Household Income (RM) & $4,542.86$ \\
\hline Variables & $\mathbf{N}(\%)$ \\
\hline $\begin{array}{l}\text { Gender } \\
\quad \text { Female }\end{array}$ & \\
$\quad$ Male & $17(49 \%)$ \\
\hline Variables & $18(51 \%)$ \\
\hline Race & $\mathbf{N}(\%)$ \\
$\quad$ Malay & \\
Chinese & $26(74 \%)$ \\
$\quad$ Indian & $6(17 \%)$ \\
$\quad$ Caucasian (German) & $2(6 \%)$ \\
\hline Exercise during leisure time? & $1(3 \%)$ \\
$\quad$ Yes & \\
No & $25(71 \%)$ \\
\hline
\end{tabular}

\begin{tabular}{|l|l|}
\hline Variables & $\mathbf{N}(\%)$ \\
\hline Smartphone Operating System & \\
Android & $26(74 \%)$ \\
iOS & $8(23 \%)$ \\
Others & $1(3 \%)$ \\
\hline Smartphone used for mobile gaming? & \\
Yes & $29(83 \%)$ \\
No & $6(17 \%)$ \\
\hline Variables & $\mathbf{N}(\%)$ \\
\hline Main use of smartphone & \\
Communication \& Social Media & $23(66 \%)$ \\
Entertainment \& Gaming & $7(20 \%)$ \\
Web surfing & $3(8 \%)$ \\
Business & $1(3 \%)$ \\
Reading novel & $1(3 \%)$ \\
\hline
\end{tabular}

With the IPAQ results, the respondents were further split into three sub-categories based on their PA levels: High, Moderate, and Low. The total respondents were almost evenly split into the PA level groups, leaning heavily in the Low PA level group (LG) (37\%). More males were found in the High PA LG (20\%) and Moderate 
PA LG (20\%), whereas most females are in the Low PA LG (26\%). The Malay ethnic race is mostly from the Low PA LG (29\%). iOS users were found predominantly in the High PA LG (11\%). High PA LG had the highest mobile gaming players (90\%) and also engaged more PA in the High PA LG during leisure times $(90 \%)$. The monthly household income was the highest (RM5,900.00) from the High PA LG and lowest (RM3,692.31) from the Low PA LG. Daily smartphone usage was the highest in the High PA LG (6.80 hours) and lowest in Low PA LG (4.77 hours). Daily mobile gaming was the highest in the High PA LG (1.50 hours), followed by Low PA LG (1.45 hours) then Moderate PA LG (1.06 hours). Low PA LG spent more daily sedentary times (6.75 hours), followed by High PA LG (6.45 hours) then Moderate PA LG (5.13 hours). Respondents in the High PA LG rated themselves highest at 3.60 (on a scale of up to 5) in terms of their fitness rating, followed by those from Moderate PA LG (3.17) then Low PA LG (3.15). Engaging in PA during leisure times, monthly household income status, daily smartphone use, and fitness self-rating had a direct relationship with PA level groups; i.e., the higher the PA level, the higher the occurrence/situation was found. Refer to Table 3.

Table 3. Distribution of IPAQ scores split from PA levels against sociodemographic variables, smartphone usage and physical inactivity/activity (* Direct relationship with PA levels)

\begin{tabular}{|c|c|c|c|c|}
\hline \multirow{3}{*}{\multicolumn{2}{|c|}{ Variables }} & \multicolumn{3}{|c|}{ PA Levels } \\
\hline & & Low & Moderate & High \\
\hline & & \multicolumn{3}{|l|}{$\mathbf{N}(\%)$} \\
\hline \multicolumn{2}{|c|}{ 1. Total Respondents $(N=35)$} & $13(37 \%)$ & $12(34 \%)$ & $10(29 \%)$ \\
\hline \multirow[t]{2}{*}{ 2. Gender } & Female & $9(26 \%)$ & $5(14 \%)$ & $3(9 \%)$ \\
\hline & Male & $4(11 \%)$ & $7(20 \%$ & $7(20 \%)$ \\
\hline \multirow[t]{4}{*}{ 3. Race } & Malay & $10(29 \%)$ & $9(26 \%)$ & $7(20 \%)$ \\
\hline & Chinese & $3(9 \%)$ & $1(3 \%)$ & $2(6 \%)$ \\
\hline & Indian & - & $2(6 \%)$ & - \\
\hline & Caucasian (German) & - & - & $1(3 \%)$ \\
\hline \multirow{3}{*}{$\begin{array}{l}\text { 4. Smartphone } \\
\text { Operating System }\end{array}$} & Android & $10(29 \%)$ & $10(29 \%)$ & $6(17 \%)$ \\
\hline & $i O S$ & $3(9 \%)$ & $1(3 \%)$ & $4(11 \%)$ \\
\hline & Others & - & $1(3 \%)$ & - \\
\hline \multicolumn{2}{|c|}{ 5. Playing mobile games (Yes) } & $11(85 \%)$ & $9(75 \%)$ & $9(90 \%)$ \\
\hline \multirow{2}{*}{\multicolumn{2}{|c|}{ 6. Engage in PA during leisure times (Yes)* }} & $6(46 \%)$ & $10(83 \%)$ & $9(90 \%)$ \\
\hline & & \multicolumn{3}{|l|}{ Mean } \\
\hline \multicolumn{2}{|c|}{ 7. Monthly Household Income (RM) * } & $3,692.31$ & $4,333.33$ & $5,900.00$ \\
\hline \multicolumn{2}{|c|}{ 8. Daily smartphone use (hours) * } & 4.77 & 6.00 & 6.80 \\
\hline \multicolumn{2}{|c|}{ 9. Daily mobile gaming use (hours) } & 1.45 & 1.06 & 1.50 \\
\hline \multicolumn{2}{|c|}{ 10. Daily sedentary time (hours) } & 6.75 & 5.13 & 6.45 \\
\hline \multicolumn{2}{|c|}{ 11. Fitness Self-rating * } & 3.15 & 3.17 & 3.60 \\
\hline
\end{tabular}

\subsection{Discussion}

Respondents from the High PA LG were found to be heavy mobile phone users. Their average monthly household income was also the highest compared to the other groups. The High PA LG also showed a great extent to engage PA in leisure time and rated themselves highly on their fitness. For all these situations, the relationship progressively increases from Low, Moderate to High PA LGs, and these data are represented in the following graph (Refer Figure 1).

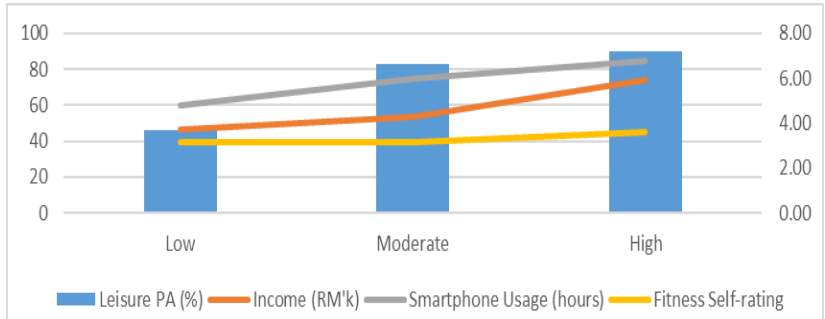

Figure 1. Direct Relationship between PA level and Leisure PA (\%), Income (RM'k), Smartphone Usage (hours) and Fitness Self-rating 
Based on the data results from Table 3, three distinct UPs were created using the IPAQ's PA levels as the basis of difference. The UPs were derived from the data extracted in the user study, and the following three characters were created: Alia (representing Low PA level), Baharin (representing Moderate PA level) and Kelvin (representing High PA level). Refer Table 4.

Table 4. User Personas' Background

\begin{tabular}{|c|c|c|c|}
\hline $\begin{array}{ll}\text { Variables } & \text { PA Level } \\
\end{array}$ & Low & Moderate & High \\
\hline Representative & Alia & Baharin & Kelvin \\
\hline Gender & Female & Male & Male \\
\hline Age & 20 & 21 & 22 \\
\hline Race & Malay & Malay & Chinese \\
\hline Income Status (RM) & RM3,692.31 & RM4,333.33 & RM5,900.00 \\
\hline Smartphone O.S. & Android & Android & $\mathrm{iOS}$ \\
\hline
\end{tabular}

Character descriptions, along with their motivations and expectations:

- Alia does little PA. As she needs to supplement her income, Alia works part-time at her local convenience store's cash register. She uses her smartphone to communicate with her friends on social media and play mobile games whenever possible, when at leisure. Alia is concerned with her lack of fitness, but she needs to work during her free time to supplement her income.

- Baharin's daily PA is achieved through his daily walks to campus. He uses his smartphone mainly to communicate with his peers and less with gaming. He finds the daily walks beneficial to his fitness but has an interest in doing structured fitness routines, such as CrossFit, if he has the time and funds.

- Kelvin leads an active lifestyle. He enjoys running and can afford the latest mobile gadgets to keep track of his daily PAs. His smartphone is used for entertainment and keeping up to date with his work. Though Kelvin is mostly sedentary, he can manage his time well thus including PAs in his daily routines.

For each UP, the factors that influence their sedentary lifestyles are the type of PA and smartphone functions (tracking) for Kelvin; financial constraints, and time management for both Baharin and Alia. The common factor between these user personas is that the use of smartphones is crucial as a management tool for their daily use even during leisure times, thus having a high probability of being mostly sedentary given the activity performed during those times.

\section{Conclusion}

Three user personas were derived from this user study, representing the Malaysian GenZ undergraduates in terms of smartphone usage and their daily physical activities. The user personas are known as Alia, Baharin, and Kelvin, representing the respondents' PA LGs: Low, Moderate, and High, respectively. The factors that influence these youths' daily PA are smartphone functions, financial constraints, time management, and communications. The High PA level group respondents are found to be able to manage their fitness activities during leisure times, even when being highly sedentary themselves. Thus, to assist the development process of a mobile exergame design model for these youths, efforts would concentrate on the Low and Moderate PA level groups' influencing factors: time management, financial constraints, and communications.

Future work could include more respondents from the university as this user study representation is only on a sample obtained from undergraduates from one faculty. Additional questions can be added to query on the types of motivation suitable for use in encouraging PA for the Malaysian GenZ undergraduates.

\section{Acknowledgment}

We thank the Research Management Centre (RMC), Universiti Putra Malaysia (UPM), Malaysia, for funding this paper through its 'Geran Putra' grant initiative program (GP/2018/9649800) and our respondents who consented to participate in the survey. Ethics approval was obtained from the Ethics Committee for Research Involving Human Subjects (JKEUPM), UPM, Malaysia; reference: UPM/TNCPI/RMC/JKEUPM/1.4.18.2.

\section{References}

1. Barkley, J. E., Lepp, A., \& Salehi-esfahani, S. (2016). College Students' Mobile Telephone Use Is Positively Associated With Sedentary Behavior. American Journal of Lifestyle Medicine, 10(6), 437441.

2. Biber, D. (2013). Attraction to Physical Activity of Generation Z: A Mixed Methodological 
Approach. Open Journal of Preventive Medicine, 3(3), 310-319.

3. Craig, C. L., Marshall, A. L., Sjöström, M., Bauman, A. E., Booth, M. L., Ainsworth, B. E., Pratt, M., Ekelund, U., Yngve, A., Sallis, J. F., \& Oja, P. (2003). International physical activity questionnaire: 12-Country reliability and validity. Medicine and Science in Sports and Exercise, 35(8), 1381-1395.

4. Garber, C. E., Blissmer, B., Deschenes, M. R., Franklin, B. A., Lamonte, M. J., Lee, I. M., Nieman, D. C., \& Swain, D. P. (2011). Quantity and quality of exercise for developing and maintaining cardiorespiratory, musculoskeletal, and neuromotor fitness in apparently healthy adults: Guidance for prescribing exercise. Medicine and Science in Sports and Exercise, 43(7), 1334-1359.

5. Han, L., Pan, Z., Zhang, M., \& Tian, F. (2016). A Pleasurable Persuasive Model for E-fitness System. International Conference on Cyberworlds, 89-96.

6. Hashim, H. L., Kamaruddin, A., \& Jantan, A. H. (2019). The mobile exergames design model to encourage physical activity for sedentary generation Z. ACM International Conference Proceeding Series, 137-141.

7. Hashim, H. L., Kamaruddin, A., Wirza Rahmat, R. O., \& Hazri Jantan, A. (2018). Design Models for Mobile Augmented Reality Exergames: State-of-the-Art Review. International Journal of Engineering \& Technology, 7, 445-451.

8. Haskell, W. L., Lee, I. M., Pate, R. R., Powell, K. E., Blair, S. N., Franklin, B. A., MacEra, C. A., Heath, G. W., Thompson, P. D., \& Bauman, A. (2007). Physical activity and public health: Updated recommendation for adults from the American College of Sports Medicine and the American Heart Association. Medicine and Science in Sports and Exercise, 39(8), 1423-1434.

9. Hussain, A., Mkpojiogu, E.O.C., Kamal, F.M. (2016). A systematic review on usability evaluation methods for m-commerce apps. Journal of Telecommunication, Electronic and Computer Engineering, 8 (10), pp. 29-34.

10. Hussain, A., Leong, C.C., Puteh, N., Zaaba, Z.F. (2019). Requirement model of conference management system: A mobile app for creating and managing conference paper. International Journal of Interactive Mobile Technologies, 13 (11), pp. 197-212.

11. Júdice, P. B., Hamilton, M. T., Sardinha, L. B., Zderic, T. W., \& Silva, A. M. (2016). What is the metabolic and energy cost of sitting, standing and sit/stand transitions? European Journal of Applied Physiology, 116(2), 263-273.

12. Lian, T. C., Bonn, G., Han, Y. S., Choo, Y. C., \& Piau, W. C. (2016). Physical activity and its correlates among adults in Malaysia: A cross-sectional descriptive study. PLoS ONE, 11(6), 1-14.

13. Marquet, O., Alberico, C., \& Hipp, A. J. (2018). Pokémon GO and physical activity among college students. A study using Ecological Momentary Assessment. Computers in Human Behavior, 81, 215222.

14. Mueller, F. F., Khot, R. A., Gerling, K., \& Mandryk, R. (2016). Exertion Games. Foundations and Trends® in Human-Computer Interaction, 10(1), 1-86.

15. National Health and Morbidity Survey. (2015). National Health and Morbidity Survey 2015 (NHMS 2015). Vol. II: Non-Communicable Diseases, Risk Factors \& Other Health Problems. Kuala Lumpur, Malaysia, 1-291.

16. Network, S. B. R. (2013). Letter to the Editor: Standardised use of the terms "sedentary" and "sedentary behaviours." Mental Health and Physical Activity, 6(1), 55-56.

17. Nigg, C. R., Mateo, D. J., \& An, J. (2017). Pokémon GO may increase physical activity and decrease sedentary behaviors. American Journal of Public Health, 107(1), 37-38.

18. Ozkan, M., \& Solmaz, B. (2015). Mobile Addiction of Generation Z and its Effects on their Social Lifes. Procedia - Social and Behavioral Sciences, 205(May), 92-98.

19. Piette, J. D., List, J., Rana, G. K., Townsend, W., Striplin, D., \& Heisler, M. (2015). Mobile health devices as tools for worldwide cardiovascular risk reduction and disease management. Circulation, 132(21), 2012-2027.

20. Pruitt, J., \& Adlin, T. (2006). The Persona Lifecycle: Keeping People in Mind Throughout Product Design. In Morgan Kaufmann.

21. Sharp, H., Rogers, Y., \& Preece, J. (2019). Interaction Design: Beyond Human-Computer Interaction. 5th Edition (Fifth). John Wiley \& Sons, Inc.

22. Swift, D. L., Johannsen, N. M., Lavie, C. J., Earnest, C. P., \& Church, T. S. (2014). The role of exercise and physical activity in weight loss and maintenance. Progress in Cardiovascular Diseases, 56(4), 441-447.

23. T. Padmapriya \& S.V. Manikanthan, "Retracted: Security and Routing protocol for 5G wireless mobile networks". IJIMT, 2020.

24. Wang, A. I., Hagen, K., \& Høivik, T. (2018). Evaluation of the Game Exermon - A Strength Exergame Inspired by Pokémon Go. Advances in Computer Entertainment, 7624, 384-405. 